\title{
Nonlinear Behavior in a Piezoelectric Resonator: A Method of Analysis
}

\author{
José E. García, Rafel Pérez, Alfons Albareda, and Enric Minguella
}

\begin{abstract}
Theories used for understanding nonlinear behavior of piezoelectric resonators are usually only valid for a given range of amplitudes. Thus, important discrepancies can sometimes be observed between theory and experiment. In this work, a simplified model of the resonator is assumed in order to extend the analysis of nonlinear behavior to any kind of nonlinear function, without a significant increase of mathematical complexity. Nevertheless, nonlinearities are considered to be weak enough to be taken as perturbations. An asymptotic method is used to obtain the first and second order perturbations of the response to an harmonic excitation applied to the system, and each one is separated into Fourier series. Nonlinearity is described by two functions- $\Phi(\mathrm{S}, \mathrm{D}, \dot{\mathrm{S}}, \dot{\mathrm{D}})$ and $\Psi(\mathrm{S}, \mathrm{D}, \dot{\mathrm{S}}, \dot{\mathrm{D}})$ - that must be added to the constitutive equations that give $T$ and $E$ as functions of $\mathrm{S}$ and $\mathrm{D}$. These functions can be split into their symmetrical and antisymmetrical parts, which have different incidence over the perturbation terms. In order to simplify the problem, no mechanical excitation is considered, the electrical one is taken as strictly harmonic, and the current rather than the e.m.f. is taken as initial data. As an application example, this method is applied in order to find the second harmonic generation for a particular kind of nonlinearity.
\end{abstract}

\section{INTRODUCTION}

$\mathrm{N}$ ONLINEAR effects (such as harmonic generation, decrease of quality factor, or shift of resonant frequency) appear when a high strain level is applied to a piezoelectric ceramic resonator. Near the resonance frequency, all these effects can be correlated to the motional current $I_{m o t}$, which is related to the strain amplitude experienced by the ceramic material.

Most of the theories [1]-[5] accept as a basis the power series development of the energy, which means that most of the previous effects must be proportional to the square of the oscillation amplitude. Obviously, this is true for sufficiently low strain levels.

Unfortunately, experimental results often show that this prediction is not valid for moderate levels, so these models often provide only very rough approximations [6]. In practice, some phenomena cannot be adequately explained by the usual theories. We know, for example, that for some materials experimental data fit $I_{m o t}$ or $I_{m o t}^{\frac{3}{2}}$ better than

Manuscript received June 8, 1999; accepted December 21, 1999. This work has been possible thanks to the project TAP98-0911C03-02 from CICYT, Spain. This work was presented in part at the Eleventh IEEE ISAF, Montreux, 1998.

The authors are with the Departament de Física Aplicada, Universitat Politecnica de Catalunya, 08034 Barcelona, Spain (e-mail: rafel@fa.upc.es). the $I_{m o t}^{2}$ predicted by the theory. Moreover, if these effects are seen as an increase of the electrical impedance of the resonator [4], the relation defined as $m=\Delta X / \Delta R$ between its real $\Delta R(I)$ and imaginary $\Delta X(I)$ parts is a constant in some materials, which shows that their dependence with $I$ has the same form in spite of not conforming to the expected behavior. In addition, comparisons of resonators with the same geometry but of different material show a correlation between coefficient $m$ and quality factor $Q$, so that in high quality materials the frequency shift, described by $\Delta X(I)$, is stronger than the decrease of the quality factor, described by $\Delta R(I)$.

Because of this, theories based upon power series development of nonlinear terms seem to be exceedingly restrictive, encouraging us to try to develop a method that can accept any type of nonlinear function and enabling the model to fit experimental results in the first or second approximations.

In order to develop such a method, this work is structured in three parts, based upon three different models. In the first part, an oversimplified nonlinear model is developed in which the system is defined by a single variable, and the nonlinearity can be expressed by any kind of function. Some restrictions are imposed to make the calculus converge quickly by minimizing the number of iterations. The second part is based upon a linear piezoelectric model in which the state is defined by two coupled variables for describing the mechanical and the electrical behavior. In the third part, a nonlinear piezoelectric model is presented, a conjunction of the previous models in which the restrictions that allow complexity minimization and improvement of the convergence have been revised.

\section{Nonlinear Model with a Single DEGREE OF FREEDOM}

In this section, a single degree of freedom $\mathbf{u}$ is considered, so neither the fact that there are actually two coupled systems (electrical and mechanical) nor that they are distributed systems are taken into account. Consequently, the variable $\mathbf{u}$ can symbolize displacement in a mechanical system or the charge in an electrical one.

An asymptotic resolution method is used, assuming that the nonlinearity is weak, so its solution is not very different from that of the respective linear system [7].

The normalized differential equation that applies can be written as:

$$
\ddot{\mathbf{u}}+2 \mu \dot{\mathbf{u}}+\mathbf{u}=\mathbf{F}+\epsilon \mathbf{g}(\mathbf{u}, \dot{\mathbf{u}})
$$


where the parameter $\epsilon$ is applied only to the nonlinear term, so this vanishes when $\epsilon$ tends to zero, but it is applied neither to the dissipative term nor to the external force. The nonlinear term $\mathbf{g}(\mathbf{u}, \dot{\mathbf{u}})$ depends on the phase space so the function and its first and second derivatives are zero at the origin. Thanks to this definition, the function $\mathrm{g}$ not only states elastic nonlinearities but also nonlinear losses.

In order to simplify the notation, a linear operator $G$ is defined:

$$
G \equiv \frac{d^{2}}{d t^{2}}+2 \mu \frac{d}{d t}+1
$$

With this operator, the differential equation (1) can be written as:

$$
G(\mathbf{u})=\mathbf{F}+\epsilon \mathbf{g}(\mathbf{u}, \dot{\mathbf{u}})
$$

Because nonlinearity can be expressed as a scalar field in the phase space, and the periodic evolution of the system is given by a closed orbit in that space, the value of $\mathbf{g}$ at each moment can be found. It must be considered that the actual orbit $\mathbf{u}$ differs slightly from the linear one $\mathbf{u}_{0}$, so it can be written as:

$$
\mathbf{u}=\mathbf{u}_{0}+\epsilon \mathbf{u}_{1}+\epsilon^{2} \mathbf{u}_{2}
$$

Odd harmonics are seen as centrosymmetric deviations of the orbit, and even harmonics are shown as noncentrosymmetric perturbations. We will assume that $\mathbf{u}_{0}$, the linear part of $\mathbf{u}$, which is sinusoidal, is fixed a priori in amplitude $A$ and phase:

$$
\mathbf{u}_{0}=A \cos \omega_{o} t
$$

so the perturbation functions $\mathbf{u}_{1}$ and $\mathbf{u}_{2}$ do not contain fundamental harmonic.

We also will assume that the external force is strictly harmonic, but its amplitude is not fixed, because it depends on the value of $A$ and the strength of the nonlinearity:

$$
\mathbf{F}=\Re\left[\left(F_{0}^{I}+\epsilon F_{1}^{I}+\epsilon^{2} F_{2}^{I}\right) e^{j \omega_{o} t}\right]
$$

The nonlinear effects produced at the fundamental frequency are described by the perturbations of the force $\mathbf{F}$, and those produced at harmonic frequencies are described by the perturbations of $\mathbf{u}$.

Because the actual trajectory $(\mathbf{u}, \dot{\mathbf{u}})$ is not very different from the linear one $\left(\mathbf{u}_{0}, \dot{\mathbf{u}}_{0}\right)$, the function $\mathbf{g}(\mathbf{u}, \dot{\mathbf{u}})$ can be developed in power series of the parameter $\epsilon$ :

$$
\begin{aligned}
\mathbf{g}(\mathbf{u}, \dot{\mathbf{u}})= & \mathbf{g}\left(\mathbf{u}_{0}, \dot{\mathbf{u}}_{0}\right)+ \\
& \epsilon\left[\mathbf{u}_{1} \frac{\partial \mathbf{g}}{\partial \mathbf{u}}\left(\mathbf{u}_{0}, \dot{\mathbf{u}}_{0}\right)+\dot{\mathbf{u}}_{1} \frac{\partial \mathbf{g}}{\partial \dot{\mathbf{u}}}\left(\mathbf{u}_{0}, \dot{\mathbf{u}}_{0}\right)\right]+\mathcal{O}\left(\epsilon^{2}\right)
\end{aligned}
$$

Then, the differential equation can be detached into three equations, one for each approximation order:

$$
\begin{aligned}
G\left(\mathbf{u}_{0}\right) & =\Re\left(F_{0}^{I} e^{j \omega_{0} t}\right) \\
G\left(\mathbf{u}_{1}\right) & =\Re\left(F_{1}^{I} e^{j \omega_{0} t}\right)+\mathbf{g}\left(\mathbf{u}_{0}, \dot{\mathbf{u}}_{0}\right) \\
G\left(\mathbf{u}_{2}\right) & =\Re\left(F_{2}^{I} e^{j \omega_{0} t}\right)+\mathbf{u}_{1} \frac{\partial \mathbf{g}}{\partial \mathbf{u}}\left(\mathbf{u}_{0}, \dot{\mathbf{u}}_{0}\right) \\
& +\dot{\mathbf{u}}_{1} \frac{\partial \mathbf{g}}{\partial \dot{\mathbf{u}}}\left(\mathbf{u}_{0}, \dot{\mathbf{u}}_{0}\right) .
\end{aligned}
$$

\section{A. Detachment into Successive Approximations}

Taking into account that the system has reached a stationary state under a periodical external force, all the terms that appear are periodical functions of time, so their Fourier transforms have only terms of multiple frequency of the main frequency.

We will use the superindices: ${ }^{0}$ I II III in order to assign the complex amplitude of the respective harmonic term of a given function. We also will use these superindices to assign the respective Fourier transforms of the operator $G$.

The operator $G$ can be described by a function of the frequency:

$$
G^{n}=-\left(n \omega_{o}\right)^{2}+j\left(2 n \omega_{o}\right) \mu+1
$$

where $n$ is the number assigned to the respective harmonic.

There are other resonances not considered by this model, due to the fact that the system is actually continuous, so $G^{I I}$ and $G^{I I I}$ may be rather different from their expected expression.

Equation (8) contains only terms of fundamental frequency, and it allows to obtain $F_{0}^{I}$ in the form:

$$
F_{0}^{I}=G^{I}\left(u_{0}^{I}\right)
$$

where $u_{0}^{I}$ denotes the complex amplitude of $\mathbf{u}_{0}$.

In (9) and (10), the left-hand term does not contain fundamental frequency, and the first right-hand term has only fundamental frequency. So, in the first approximation we find the following expressions:

$$
\begin{aligned}
& F_{1}^{I}=-g^{I}\left(\mathbf{u}_{0}, \dot{\mathbf{u}}_{0}\right) \\
& G^{0}\left(u_{1}^{0}\right)=g^{0}\left(\mathbf{u}_{0}, \dot{\mathbf{u}}_{0}\right) \\
& G^{I I}\left(u_{1}^{I I}\right)=g^{I I}\left(\mathbf{u}_{0}, \dot{\mathbf{u}}_{0}\right) \\
& G^{I I I}\left(u_{1}^{I I I}\right)=g^{I I I}\left(\mathbf{u}_{0}, \dot{\mathbf{u}}_{0}\right) .
\end{aligned}
$$

For the second approximation we find:

$$
\begin{aligned}
& F_{2}^{I}=-\left[\mathbf{u}_{1} \frac{\partial \mathbf{g}}{\partial \mathbf{u}}\left(\mathbf{u}_{0}, \dot{\mathbf{u}}_{0}\right)+\dot{\mathbf{u}}_{1} \frac{\partial \mathbf{g}}{\partial \dot{\mathbf{u}}}\left(\mathbf{u}_{0}, \dot{\mathbf{u}}_{0}\right)\right]^{I} \\
& G^{0}\left(u_{2}^{0}\right)=\left[\mathbf{u}_{1} \frac{\partial \mathbf{g}}{\partial \mathbf{u}}\left(\mathbf{u}_{0}, \dot{\mathbf{u}}_{0}\right)+\dot{\mathbf{u}}_{1} \frac{\partial \mathbf{g}}{\partial \dot{\mathbf{u}}}\left(\mathbf{u}_{0}, \dot{\mathbf{u}}_{0}\right)\right]^{0} \\
& G^{I I}\left(u_{2}^{I I}\right)=\left[\mathbf{u}_{1} \frac{\partial \mathbf{g}}{\partial \mathbf{u}}\left(\mathbf{u}_{0}, \dot{\mathbf{u}}_{0}\right)+\dot{\mathbf{u}}_{1} \frac{\partial \mathbf{g}}{\partial \dot{\mathbf{u}}}\left(\mathbf{u}_{0}, \dot{\mathbf{u}}_{0}\right)\right]^{I I} \\
& G^{I I I}\left(u_{2}^{I I I}\right)=\left[\mathbf{u}_{1} \frac{\partial \mathbf{g}}{\partial \mathbf{u}}\left(\mathbf{u}_{0}, \dot{\mathbf{u}}_{0}\right)+\dot{\mathbf{u}}_{1} \frac{\partial \mathbf{g}}{\partial \dot{\mathbf{u}}}\left(\mathbf{u}_{0}, \dot{\mathbf{u}}_{0}\right)\right]^{I I I}
\end{aligned}
$$

which allow us to find the function $\mathbf{u}_{2}$ from the previous approximation. 
TABLE I

Repercussion of the Symmetrical $S$ and Antisymmetrical $A$ Terms of the Function $g(u, \dot{u})$ Over Each of the Effects

Taken Into Account, in First (Direct) and Second (Indirect) Order Approximation.

\begin{tabular}{ccccccc}
\hline DIR. & $S$ & $A$ & INDIR. & $S S$ & $A A$ & $S A$ \\
\hline$F_{1}^{I}$ & & $\mathrm{X}$ & $F_{2}^{I}$ & $\mathrm{X}$ & $\mathrm{X}$ & \\
$u_{1}^{I I I}$ & & $\mathrm{X}$ & $u_{2}^{I I I}$ & $\mathrm{X}$ & $\mathrm{X}$ & \\
$u_{1}^{I I}$ & $\mathrm{X}$ & & $u_{2}^{I I}$ & & & $\mathrm{X}$ \\
$u_{1}^{0}$ & $\mathrm{X}$ & & $u_{2}^{0}$ & & & $\mathrm{X}$ \\
\hline
\end{tabular}

\section{B. Symmetry of the Nonlinear Function}

The function $\mathbf{g}$ can be split into a symmetrical and an antisymmetrical one:

$$
\mathbf{g}(\mathbf{u}, \dot{\mathbf{u}})=\mathbf{g}_{S}(\mathbf{u}, \dot{\mathbf{u}})+\mathbf{g}_{A}(\mathbf{u}, \dot{\mathbf{u}})
$$

where:

$$
\begin{aligned}
& \mathbf{g}_{A}(\mathbf{u}, \dot{\mathbf{u}})=-\mathbf{g}_{A}(-\mathbf{u},-\dot{\mathbf{u}}) \\
& \mathbf{g}_{S}(\mathbf{u}, \dot{\mathbf{u}})=\mathbf{g}_{S}(-\mathbf{u},-\dot{\mathbf{u}})
\end{aligned}
$$

Then, owing to the symmetry of $\mathbf{u}_{0}$, the nonlinear function $\mathbf{g}_{A}\left(\mathbf{u}_{0}, \dot{\mathbf{u}}_{0}\right)$ only contains odd order harmonics, denoted as $g_{A}^{I}$ and $g_{A}^{I I I}$, whereas $\mathbf{g}_{S}\left(\mathbf{u}_{0}, \dot{\mathbf{u}}_{0}\right)$ only contains even harmonics $g_{S}^{0}$ and $g_{S}^{I I}$. Then, only the antisymmetrical term contributes to the function $F_{1}^{I}$ :

$$
\begin{aligned}
F_{1}^{I} & =-g_{A}^{I}=-\frac{\omega}{2 \pi} \int_{0}^{\frac{2 \pi}{\omega}} \mathbf{g}_{A}\left(\mathbf{u}_{0}, \dot{\mathbf{u}}_{0}\right) e^{-j \omega t} d t \\
& =-\frac{1}{2 \pi} \int_{0}^{2 \pi} g_{A}(A \cos \phi,-A \omega \sin \phi) e^{-j \phi} d \phi .
\end{aligned}
$$

We see that the even components ${ }^{0}$ and ${ }^{I I}$ of the function $u_{1}$ depend on the symmetric function, whereas the odd component ${ }^{I I I}$ depends on the antisymmetrical one:

$$
\begin{aligned}
& G^{0}\left(u_{1}^{0}\right)=\frac{1}{2 \pi} \int_{0}^{2 \pi} g_{S}(A \cos \phi,-A \omega \sin \phi) d \phi \\
& G^{I I}\left(u_{1}^{I I}\right)=\frac{1}{2 \pi} \int_{0}^{2 \pi} g_{S}(A \cos \phi,-A \omega \sin \phi) e^{-j 2 \phi} d \phi \\
& G^{I I I}\left(u_{1}^{I I I}\right)=\frac{1}{2 \pi} \int_{0}^{2 \pi} g_{A}(A \cos \phi,-A \omega \sin \phi) e^{-j 3 \phi} d \phi
\end{aligned}
$$

Otherwise, the respective derivatives with respect to $\mathbf{u}$ or $\dot{\mathbf{u}}$, have opposite symmetry. Then, in order to obtain $F_{2}^{I}$, which is antisymmetrical, the product of symmetrical $\mathbf{u}_{1}$ by derivatives of $\mathbf{g}_{S}$, in addition to the product of antisymmetrical $\mathbf{u}_{1}$ by derivatives of $\mathbf{g}_{A}$, must be considered as shown in Table I. In Table I, a cross indicates that such an effect is produced by that type ( $A$ or $S$ ) of nonlinearity, otherwise it is indicated by a blank.

If there are only direct effects, these are linear with respect to the causes $(\mathbf{g}(\mathbf{u}, \dot{\mathbf{u}}))$ that produce them. This is no longer true when indirect effects play a non-negligible role, in which case all combinations are possible.

\section{Linear System with Two Coupled DEGREeS of FrEedom}

There is an additional difficulty in applying the last model to a piezoelectric resonator, because two degrees of freedom are needed to describe it (one for the mechanical state, and one for the electrical state). In each case we must take the adequate variables: we have taken the mechanical strain $\mathbf{S}$ and electrical displacement $\mathbf{D}$, so the state of the system is defined without ambiguity for each pair of values $(\mathbf{S}, \mathbf{D})$. The study is restricted to a one-dimensional case, so thickness resonators only are considered. Although the variables $\mathbf{S}$ and $\mathbf{D}$ are, in general, functions of time and space, we restricted our study by assuming that they are reasonably uniform; so the thickness of the transducer must be significantly less than the wavelength of the acoustical wave.

The last conditions are fulfilled by a sandwich transducer (Langevin). If it is symmetrical, only a half transducer must be studied, in which there is a node at one end (infinite mechanical impedance) and a mass at the other end, to which will be assigned a mechanical impedance $Z_{m e c}$. We shall call the total thickness and section area of the transducer $2 l$ and $W$, respectively.

If the variable $\mathbf{u}$ describes the displacement of the surface between the ceramic and the countermass, then $\mathbf{S}=\mathbf{u} / l$, assuming that $\mathbf{S}$ is uniform.

\section{A. Linear Constitutive Equations and Boundary Conditions}

Without any losses, the variables $\mathbf{S}$ and $\mathbf{D}$ are related to the mechanical and electrical "forces" $\mathbf{T}$ and $\mathbf{E}$ that are applied to the material through the constitutive equations, which can be expressed in their matricial form:

$$
\left(\begin{array}{cc}
c^{D} & -h \\
-h & \frac{1}{\epsilon^{s}}
\end{array}\right)\left(\begin{array}{l}
\mathbf{S} \\
\mathbf{D}
\end{array}\right)=\left(\begin{array}{l}
\mathbf{T} \\
\mathbf{E}
\end{array}\right)
$$

As $\mathbf{D}$ must be uniform due to the Gauss law, and $\mathbf{S}$ is assumed to be uniform, $\mathbf{T}$ and $\mathbf{E}$ are also assumed to be uniform. The field $\mathbf{E}$ is related to the voltage applied to the piezoelectric $\mathbf{V}=\mathbf{E} l$.

If there are losses, a matricial term must be added to (27), which includes not only mechanical losses but also electrical and piezoelectric ones:

$$
\left(\begin{array}{cc}
c^{D} & -h \\
-h & \frac{1}{\epsilon^{s}}
\end{array}\right)\left(\begin{array}{l}
\mathbf{S} \\
\mathbf{D}
\end{array}\right)+\left(\begin{array}{cc}
\alpha_{m} & \alpha_{p} \\
\alpha_{p} & \alpha_{e}
\end{array}\right)\left(\begin{array}{c}
\dot{\mathbf{S}} \\
\dot{\mathbf{D}}
\end{array}\right)=\left(\begin{array}{l}
\mathbf{T} \\
\mathbf{E}
\end{array}\right)
$$

This expression can be written in a compact form:

$$
\left(\begin{array}{cc}
c^{D}+\alpha_{m} \frac{\partial}{\partial t}-h+\alpha_{p} \frac{\partial}{\partial t} \\
-h+\alpha_{p} \frac{\partial}{\partial t} \frac{1}{\epsilon^{s}}+\alpha_{e} \frac{\partial}{\partial t}
\end{array}\right)\left(\begin{array}{l}
\mathbf{S} \\
\mathbf{D}
\end{array}\right)=\left(\begin{array}{l}
\mathbf{T} \\
\mathbf{E}
\end{array}\right) .
$$

When the linearity of the system is assumed, each harmonic component can be treated separately, so a complex formulation is advantageous, and complex amplitudes can 
be used instead of time functions. For a periodic excitation of fundamental frequency $\omega_{o}$, the response also will be periodical, and we will have only frequencies multiples of $\omega_{o}$. We can write:

$$
\left(\begin{array}{l}
\mathbf{S} \\
\mathbf{D}
\end{array}\right)=\Re\left\{\sum_{n}\left[\left(\begin{array}{c}
S^{n} \\
D^{n}
\end{array}\right) e^{j n \omega_{o} t}\right]\right\} .
$$

For a single frequency (harmonic excitation), (29) can be written as:

$$
\left(\begin{array}{cc}
c^{D}\left(1+j \frac{1}{Q_{m}}\right) & -h\left(1+j \frac{1}{Q_{p}}\right) \\
-h\left(1+j \frac{1}{Q_{p}}\right) & \frac{1}{\epsilon^{s}}\left(1+j \frac{1}{Q_{e}}\right)
\end{array}\right)\left(\begin{array}{c}
S \\
D
\end{array}\right)=\left(\begin{array}{c}
T \\
E
\end{array}\right)
$$

where the mechanical, electrical, and piezoelectric losses are expressed in terms of $Q_{m}, Q_{e}$, and $Q_{p}$. The countermass $M$ is modeled as a mechanical impedance $Z_{\text {mec }}$ connected to the piezoelectric piece. Then, if we assume that there is no mechanical excitation:

$$
T=-\frac{Z_{m e c}}{W} \dot{u}
$$

So,

$$
T=-j \omega \frac{l}{W} Z_{m e c} S
$$

In a similar way, if the transducer is electrically connected to a generator of electromotive force $\mathcal{E}$ with an electrical impedance $Z_{e l}$, then:

$$
\mathcal{E}=V+I Z_{e l}
$$

and,

$$
E=\frac{\mathcal{E}}{l}-j \omega \frac{W}{l} Z_{e l} D
$$

The (32) and (33) can be joined in a matricial form:

$$
\left(\begin{array}{c}
T \\
E
\end{array}\right)=\left(\begin{array}{cc}
-j \omega \frac{l}{W} Z_{m e c} & 0 \\
0 & -j \omega \frac{W}{l} Z_{e l}
\end{array}\right)\left(\begin{array}{c}
S \\
D
\end{array}\right)+\left(\begin{array}{c}
0 \\
\frac{1}{l} \mathcal{E}
\end{array}\right)
$$

\section{B. Linear Operator Matrix. Linear Solution}

As we have seen, vector $(T, E)$ can be related to $(S, D)$ through constitutive equations, with losses included (31), and on the other side it can be expressed in terms of the boundary conditions and the applied electromotive force (34). Identifying both expressions, and arranging the boundary terms with the constitutive ones, we attain the usual format of a differential equation system, in which one of the independent terms (the mechanical excitation) is null. So:

$$
\left(\begin{array}{ll}
L_{11} & L_{12} \\
L_{21} & L_{22}
\end{array}\right)\left(\begin{array}{c}
S \\
D
\end{array}\right)=\left(\begin{array}{c}
0 \\
\frac{1}{l} \mathcal{E}
\end{array}\right)
$$

where $L_{11}, L_{12}, L_{21}$, and $L_{22}$ symbolize linear operators applied to the variables $S$ and $D$. The operator matrix
$L_{i j}(\omega)$ can be written as:

$(L)=\left(\begin{array}{cc}c^{D}+j\left(\omega \frac{l}{W} Z_{m e c}+\frac{c^{D}}{Q_{m}}\right) & -h+j \frac{1}{Q_{p}} \\ -h+j \frac{1}{Q_{p}} & \frac{1}{\epsilon^{s}}+j\left(\omega \frac{W}{l} Z_{e l}+\frac{1}{\epsilon^{s} Q_{e}}\right)\end{array}\right)(36)$

The (35) can be solved for each frequency by the use of complex variables. In this case, we can obtain $S$ and $D$ from the value of $\mathcal{E}$ without any other difficulty than the inversion of the operator matrix, in which each coefficient is a complex function of the frequency.

In the linear case, it may be convenient to approach the problem in the following way: which voltage must be applied (in amplitude and phase) in order to reach a given current? In this case, the initial data is $D$ (because $I=$ $j \omega W D)$ so, applying the first row of operators, we obtain:

$$
S=-\frac{L_{12}}{L_{11}} D
$$

and from (37) we can obtain the modulus and phase of $S$, that can be used to calculate the complex value of $\mathcal{E}$ through the second row of the operator matrix:

$$
\mathcal{E}=\left(L_{22}-\frac{L_{12}^{2}}{L_{11}}\right) l D
$$

Then, the electromotive force $\mathcal{E}$ and the voltage $V$ can be expressed as a function of the current $I$, which yields the expression of the electrical impedance of the resonator:

$$
Z(\omega)=-j\left(L_{22}-\frac{L_{12}^{2}}{L_{11}}\right) \frac{l}{\omega W}-Z e l .
$$

\section{Nonlinear Analysis with Two Degrees of Freedom}

When a weak nonlinearity is assumed in an electromechanically coupled system, we can join the procedure described in Section II to the system stated in Section III.

As a departure point, the nonlinearity can be considered a modification of both constitutive equations through the terms $\Phi$ and $\Psi$, which are, in general, not only functions of the variables $\mathbf{S}$ and $\mathbf{D}$ but also functions of their time derivatives. Although in Section II the functions are normalized, in the present case this is no longer suitable. However, the use of the parameter $\epsilon$, which multiplies the nonlinear terms, still stands.

$$
\left(\begin{array}{cc}
c^{D}-h \\
-h \frac{1}{\epsilon^{s}}
\end{array}\right)\left(\begin{array}{c}
\mathbf{S} \\
\mathbf{D}
\end{array}\right)=\left(\begin{array}{c}
\mathbf{T} \\
\mathbf{E}
\end{array}\right)+\epsilon\left(\begin{array}{l}
\Phi(\mathbf{S}, \mathbf{D}, \dot{\mathbf{S}}, \dot{\mathbf{D}}) \\
\Psi(\mathbf{S}, \mathbf{D}, \dot{\mathbf{S}}, \dot{\mathbf{D}})
\end{array}\right)
$$

Taking into account the contribution of all frequencies involved, we obtain:

$$
\begin{aligned}
& \Re\left\{\sum_{n}\left[\left[\left(\begin{array}{cc}
L_{11}^{n} & L_{12}^{n} \\
L_{21}^{n} & L_{22}^{n}
\end{array}\right)\left(\begin{array}{c}
S^{n} \\
D^{n}
\end{array}\right)-\left(\begin{array}{c}
0 \\
\frac{1}{l} \mathcal{E}^{n}
\end{array}\right)\right] e^{j n \omega t}\right]\right\} \\
& =\epsilon\left(\begin{array}{c}
\Phi(\mathbf{S}, \mathbf{D}, \dot{\mathbf{S}}, \dot{\mathbf{D}}) \\
\Psi(\mathbf{S}, \mathbf{D}, \dot{\mathbf{S}}, \dot{\mathbf{D}})
\end{array}\right) .
\end{aligned}
$$


The expected solutions will be expressed as a power series of this parameter, leading to different levels of approximation. So the general solution, which cannot be sinusoidal, must be described as a function of time instead of a complex amplitude:

$$
\left(\begin{array}{l}
\mathbf{S} \\
\mathbf{D}
\end{array}\right)=\left(\begin{array}{l}
\mathbf{S}_{\mathbf{o}} \\
\mathbf{D}_{\mathbf{o}}
\end{array}\right)+\epsilon\left(\begin{array}{l}
\mathbf{S}_{\mathbf{1}} \\
\mathbf{D}_{\mathbf{1}}
\end{array}\right)+\epsilon^{2}\left(\begin{array}{c}
\mathbf{S}_{\mathbf{2}} \\
\mathbf{D}_{\mathbf{2}}
\end{array}\right)+\cdots
$$

where the subindices 0,1 , and 2 have been used to designate the linear, first and second approximations, respectively. Nonlinearity is assumed to be weak enough for the first term to be much greater than the other two, so in the phase map (that has to be described in a space of dimension four) the actual stationary trajectory of the system follows the expected linear trajectory $(\epsilon \rightarrow 0)$ quite closely.

This fact implies that nonlinear terms can be developed as a function of parameter $\epsilon$ :

$$
\left(\begin{array}{c}
\Phi \\
\Psi
\end{array}\right)=\left(\begin{array}{l}
\Phi_{\mathbf{o}} \\
\Psi_{\mathbf{o}}
\end{array}\right)+\epsilon\left(\begin{array}{l}
\Phi_{1} \\
\Psi_{1}
\end{array}\right)+\cdots
$$

where

$$
\begin{aligned}
& \Phi_{\mathbf{o}}=\Phi\left(\mathbf{S}_{\mathbf{o}}, \mathbf{D}_{\mathbf{o}}, \dot{\mathbf{S}}_{\mathbf{o}}, \dot{\mathbf{D}}_{\mathbf{o}}\right) \\
& \Psi_{\mathbf{o}}=\Psi\left(\mathbf{S}_{\mathbf{o}}, \mathbf{D}_{\mathbf{o}}, \dot{\mathbf{S}}_{\mathbf{o}}, \dot{\mathbf{D}}_{\mathbf{o}}\right) .
\end{aligned}
$$

In the definition of $\Phi_{1}$ and $\Psi_{1}$, we must take into account that $\Phi$ and $\Psi$ are four-variable functions:

$$
\begin{aligned}
& \Phi_{1}=\mathbf{S}_{1} \frac{\partial \Phi}{\partial \mathbf{S}}+\mathbf{D}_{1} \frac{\partial \Phi}{\partial \mathbf{D}}+\dot{\mathbf{S}}_{1} \frac{\partial \Phi}{\partial \dot{\mathbf{S}}}+\dot{\mathbf{D}}_{1} \frac{\partial \Phi}{\partial \dot{\dot{D}}} \\
& \Psi_{1}=\mathbf{S}_{1} \frac{\partial \Psi}{\partial \mathbf{S}}+\mathbf{D}_{1} \frac{\partial \Psi}{\partial \mathbf{D}}+\dot{\mathbf{S}}_{\mathbf{1}} \frac{\partial \Psi}{\partial \dot{\mathbf{S}}}+\dot{\mathbf{D}}_{1} \frac{\partial \Psi}{\partial \dot{\mathbf{D}}}
\end{aligned}
$$

As in the simple case, the functions $\Phi$ and $\Psi$ can be split into the sum of two functions, symmetrical and antisymmetrical, so,

$$
\begin{aligned}
& \Phi(\mathbf{S}, \mathbf{D}, \dot{\mathbf{S}}, \dot{\mathbf{D}})=\Phi_{\mathbf{S}}(\mathbf{S}, \mathbf{D}, \dot{\mathbf{S}}, \dot{\mathbf{D}})+\Phi_{\mathbf{A}}(\mathbf{S}, \mathbf{D}, \dot{\mathbf{S}}, \dot{\mathbf{D}}) \\
& \Psi(\mathbf{S}, \mathbf{D}, \dot{\mathbf{S}}, \dot{\mathbf{D}})=\Psi_{\mathbf{S}}(\mathbf{S}, \mathbf{D}, \dot{\mathbf{S}}, \dot{\mathbf{D}})+\Psi_{\mathbf{A}}(\mathbf{S}, \mathbf{D}, \dot{\mathbf{S}}, \dot{\mathbf{D}})
\end{aligned}
$$

where these functions satisfy:

$$
\begin{aligned}
& \Phi_{\mathbf{S}}(\mathbf{S}, \mathbf{D}, \dot{\mathbf{S}}, \dot{\mathbf{D}})=\Phi_{\mathbf{S}}(-\mathbf{S},-\mathbf{D},-\dot{\mathbf{S}},-\dot{\mathbf{D}}) \\
& \Phi_{\mathbf{A}}(\mathbf{S}, \mathbf{D}, \dot{\mathbf{S}}, \dot{\mathbf{D}})=-\Phi_{\mathbf{A}}(-\mathbf{S},-\mathbf{D},-\dot{\mathbf{S}},-\dot{\mathbf{D}})
\end{aligned}
$$

Due to the symmetry of $\left(\mathbf{S}_{\mathbf{o}}, \mathbf{D}_{\mathbf{o}}\right)$, the functions $\Phi_{\mathbf{A}}$ and $\Psi_{\mathbf{A}}$ have only odd harmonics, whereas $\Phi_{\mathbf{S}}$ and $\Psi_{\mathbf{S}}$ have only even harmonics, with similar results to the simple case. However, the fact that $\Phi$ is symmetrical is not enough to null the term $\mathcal{E}_{1}^{I}$, because this requires that $\Psi$ also be symmetrical.

Because the overall problem can be stated in multiple forms, we must assume some arbitrary restrictions, which have been chosen for the problem to be solved with a minimum degree of complexity. We will assume that there is no mechanical excitation, and that the electrical excitation is strictly sinusoidal. Furthermore, we will assume that the amplitude of the current at fundamental frequency is known, so no perturbation is assigned to $D$ at this frequency; then the terms $\mathbf{D}_{\mathbf{1}}$ and $\mathbf{D}_{\mathbf{2}}$ have no fundamental frequency terms, only harmonics. In spite of these restrictions, the function $\mathbf{S}$ has no restriction in its approximations.

By applying the developments (42) and (43), and a development similar to (6) for the electromotive force $\mathcal{E}$, the equation system that governs the nonlinear behavior can be developed as follows:

$$
\begin{array}{r}
\Re\left\{\sum _ { n } \left[\left[\left(\begin{array}{ll}
L_{11}^{n} & L_{12}^{n} \\
L_{21}^{n} & L_{22}^{n}
\end{array}\right)\left(\begin{array}{c}
S_{o}^{n} \\
D_{o}^{n}
\end{array}\right)\right.\right.\right. \\
+\epsilon\left(\begin{array}{cc}
L_{11}^{n} & L_{12}^{n} \\
L_{21}^{n} & L_{22}^{n}
\end{array}\right)\left(\begin{array}{c}
S_{1}^{n} \\
D_{1}^{n}
\end{array}\right)+\epsilon^{2}\left(\begin{array}{ll}
L_{11}^{n} & L_{12}^{n} \\
L_{21}^{n} & L_{22}^{n}
\end{array}\right)\left(\begin{array}{c}
S_{2}^{n} \\
D_{2}^{n}
\end{array}\right) \\
\left.\left.\left.-\left(\begin{array}{c}
0 \\
\frac{1}{l} \mathcal{E}_{o}^{n}
\end{array}\right)+\epsilon\left(\begin{array}{c}
0 \\
\frac{1}{l} \mathcal{E}_{1}^{n}
\end{array}\right)+\epsilon^{2}\left(\begin{array}{c}
0 \\
\frac{1}{l} \mathcal{E}_{2}^{n}
\end{array}\right)\right] e^{j n \omega_{o} t}\right]\right\} \\
=\epsilon\left(\begin{array}{c}
\Phi_{\mathbf{o}} \\
\Psi_{\mathbf{o}}
\end{array}\right)+\epsilon^{2}\left(\begin{array}{l}
\Phi_{\mathbf{1}} \\
\Psi_{\mathbf{1}}
\end{array}\right)+\mathcal{O}\left(\epsilon^{3}\right)
\end{array}
$$

where the expressions related to each level of approximation can be solved separately, taking the result of each iteration as data for the development of the following one.

\section{A. Linear Approximation}

It can be obtained as described in (37) and (38), and $S_{o}$ y $\mathcal{E}_{o}$ can be written in the form :

$$
\begin{gathered}
S_{o}=-\frac{L_{12}^{I}}{L_{11}^{I}} D_{o} \\
\mathcal{E}_{o}=\left(L_{22}^{I}-\frac{L_{12}^{I^{2}}}{L_{11}^{I}}\right) l D_{o} .
\end{gathered}
$$

\section{B. First Approximation}

Taking the terms on $\epsilon$ in development (48), we can write:

$$
\begin{aligned}
& \Re\left\{\sum_{n}\left[\left[\left(\begin{array}{cc}
L_{11}^{n} & L_{12}^{n} \\
L_{21}^{n} & L_{22}^{n}
\end{array}\right)\left(\begin{array}{c}
S_{1}^{n} \\
D_{1}^{n}
\end{array}\right)-\left(\begin{array}{c}
0 \\
\frac{1}{l} \mathcal{E}_{1}^{n}
\end{array}\right)\right] e^{j n \omega_{o} t}\right]\right\} \\
& =\left(\begin{array}{l}
\Phi_{\mathbf{o}}\left(\mathbf{S}_{\mathbf{o}}, \mathbf{D}_{\mathbf{o}}, \dot{\mathbf{S}}_{\mathbf{o}}, \dot{\mathbf{D}_{\mathbf{o}}}\right) \\
\Psi_{\mathbf{o}}\left(\mathbf{S}_{\mathbf{o}}, \mathbf{D}_{\mathbf{o}}, \dot{\mathbf{S}_{\mathbf{o}}}, \dot{\mathbf{D}_{\mathbf{o}}}\right)
\end{array}\right) .
\end{aligned}
$$

We must first calculate the nonlinear term over the linear trajectory, which is treated as an independent term because it depends only on the previous iteration, and then develop it in Fourier series. Due to the restrictions imposed, the term of fundamental frequency requires a different treatment from the others.

At fundamental frequency:

$$
\left(\begin{array}{ll}
L_{11}^{I} & L_{12}^{I} \\
L_{21}^{I} & L_{22}^{I}
\end{array}\right)\left(\begin{array}{c}
S_{1}^{I} \\
0
\end{array}\right)=\left(\begin{array}{c}
0 \\
\frac{1}{l} \mathcal{E}_{1}^{I}
\end{array}\right)+\left(\begin{array}{c}
\Phi_{o}^{I} \\
\Psi_{o}^{I}
\end{array}\right)
$$

from where it is deduced that

$$
S_{1}^{I}=\frac{1}{L_{11}^{I}} \Phi_{o}^{I}
$$




$$
\mathcal{E}_{1}^{I}=l L_{21}^{I} S_{1}^{I}-l \Psi_{o}^{I}
$$

Furthermore, for the other frequencies we get:

$$
\left(\begin{array}{ll}
L_{11}^{n} & L_{12}^{n} \\
L_{21}^{n} & L_{22}^{n}
\end{array}\right)\left(\begin{array}{c}
S_{1}^{n} \\
D_{1}^{n}
\end{array}\right)=\left(\begin{array}{c}
0 \\
0
\end{array}\right)+\left(\begin{array}{c}
\Phi_{o}^{n} \\
\Psi_{o}^{n}
\end{array}\right)
$$

so:

$$
\left(\begin{array}{c}
S_{1}^{n} \\
D_{1}^{n}
\end{array}\right)=\left(L^{n}\right)^{-1}\left(\begin{array}{c}
\Phi_{o}^{n} \\
\Psi_{o}^{n}
\end{array}\right)
$$

\section{Second Approximation}

Selecting the terms on $\epsilon^{2}$ from development (48), we have:

$$
\begin{aligned}
& \Re\left\{\sum_{n}\left[\left[\left(\begin{array}{cc}
L_{11}^{n} & L_{12}^{n} \\
L_{21}^{n} & L_{22}^{n}
\end{array}\right)\left(\begin{array}{c}
S_{2}^{n} \\
D_{2}^{n}
\end{array}\right)-\left(\begin{array}{c}
0 \\
\frac{1}{l} \mathcal{E}_{2}^{n}
\end{array}\right)\right] e^{j n \omega_{o} t}\right]\right\} \\
& =\left(\begin{array}{l}
\Phi_{1}\left(\mathbf{S}_{1}, \mathbf{D}_{1}, \dot{\mathbf{S}_{1}}, \dot{\mathbf{D}_{1}}\right) \\
\Psi_{1}\left(\mathbf{S}_{1}, \mathbf{D}_{1}, \dot{\mathbf{S}_{1}}, \dot{\mathbf{D}_{1}}\right)
\end{array}\right) \text {. }
\end{aligned}
$$

The perturbations $\Phi_{1}$ y $\Psi_{1}$ can be calculated, following (45), from the harmonic components of the first iteration; they also are developed in Fourier series. The fundamental frequency also receives a different treatment.

At fundamental frequency:

$$
\left(\begin{array}{ll}
L_{11}^{I} & L_{12}^{I} \\
L_{21}^{I} & L_{22}^{I}
\end{array}\right)\left(\begin{array}{c}
S_{2}^{I} \\
0
\end{array}\right)=\left(\begin{array}{c}
0 \\
\frac{1}{l} \mathcal{E}_{2}^{I}
\end{array}\right)+\left(\begin{array}{c}
\Phi_{1}^{I} \\
\Psi_{1}^{I}
\end{array}\right)
$$

so:

$$
\begin{gathered}
S_{2}^{I}=\frac{1}{L_{11}^{I}} \Phi_{1}^{I} \\
\mathcal{E}_{2}^{I}=l L_{21}^{I} S_{2}^{I}-l \Psi_{1}^{I} .
\end{gathered}
$$

For the other frequencies we have:

$$
\left(\begin{array}{ll}
L_{11}^{n} & L_{12}^{n} \\
L_{21}^{n} & L_{22}^{n}
\end{array}\right)\left(\begin{array}{c}
S_{2}^{n} \\
D_{2}^{n}
\end{array}\right)=\left(\begin{array}{c}
0 \\
0
\end{array}\right)+\left(\begin{array}{c}
\Phi_{1}^{n} \\
\Psi_{1}^{n}
\end{array}\right)
$$

that is:

$$
\left(\begin{array}{c}
S_{2}^{n} \\
D_{2}^{n}
\end{array}\right)=\left(L^{n}\right)^{-1}\left(\begin{array}{c}
\Phi_{1}^{n} \\
\Psi_{1}^{n}
\end{array}\right)
$$

\section{Analysis of Second Harmonic Generation}

As an example of an application of the method, the study of second harmonic generation in a Langevin transducer is proposed, in a first approximation, when a harmonic voltage excitation is applied at the resonant frequency.

In order to reduce the complexity of this example, we consider that the system has mechanical losses, but no dielectric nor piezoelectric ones, and that the quality factor is enough high to allow some approximations. We suppose that the functions $\Phi_{\mathrm{S}}$ y $\Psi_{\mathrm{S}}$ are not null, otherwise second harmonic cannot be produced. We also assume that the electrical impedance of the generator is nearly null, not only at fundamental frequency but also at double frequency.

\section{A. Linear Analysis}

For an harmonic excitation, the impedance associated to the countermass is:

$$
Z_{m e c}=j \omega_{o} M
$$

So the linear operator matrix (36) can be written as:

$$
\left(L^{I}\right)=\left(\begin{array}{cc}
c^{D}\left(1-\frac{\omega_{o}^{2}}{\omega_{a}^{2}}+j \frac{1}{Q_{m}}\right) & -h \\
-h & \frac{1}{\epsilon^{s}}
\end{array}\right)
$$

where $\omega_{a}$ is the antiresonance frequency:

$$
\omega_{a}^{2}=\frac{c^{D} W}{M l} .
$$

At resonance, if there are no losses, it is fulfilled for (35) that:

$$
\left(L^{I}\right)\left(\begin{array}{c}
S_{o} \\
D_{o}
\end{array}\right)=\left(\begin{array}{c}
0 \\
0
\end{array}\right)
$$

which, by solving (66), leads to:

$$
\left(\frac{\omega_{o}}{\omega_{a}}\right)^{2}=1-k^{2} \equiv \gamma
$$

where $\mathrm{k}$ is the effective electromechanical coupling factor:

$$
k^{2} \equiv \frac{h^{2} \epsilon^{s}}{c^{D}} .
$$

By the inclusion of losses, we have:

$$
\left(\begin{array}{cc}
c^{D}\left(1-\gamma+j \frac{1}{Q_{m}}\right) & -h \\
-h & \frac{1}{\epsilon^{s}}
\end{array}\right)\left(\begin{array}{c}
S_{o} \\
D_{o}
\end{array}\right)=\left(\begin{array}{c}
0 \\
\frac{1}{l} \mathcal{E}_{o}
\end{array}\right)
$$

and, from (49) and (50) we obtain:

$$
\begin{gathered}
S_{o}=\frac{1}{h \epsilon^{s}}\left(1-j \frac{1}{Q_{m}(1-\gamma)}\right) D_{o} \\
\mathcal{E}_{o}=j \frac{l}{\epsilon^{s}} \frac{1}{Q_{m}(1-\gamma)} D_{o} .
\end{gathered}
$$

At the resonance frequency, the electrical impedance of the resonator (39) can be written as a function of the mean impedance $\tilde{Z}$, whose value is that of the capacitor $C_{o}=\epsilon^{s} W / l$ at that frequency:

$$
Z=\frac{\tilde{Z}}{Q_{m}(1-\gamma)} .
$$




\section{B. Achieving the Second Harmonic}

Assuming that the model used to describe the linear operator matrix at the frequency $\omega_{o}$ is also valid at double frequency, we can write that:

$$
\left(L^{I I}\right)=\left(\begin{array}{cc}
c^{D}\left(1-4 \gamma+j \frac{1}{Q_{m}}\right) & -h \\
-h & \frac{1}{\epsilon^{s}}
\end{array}\right) .
$$

Once matrix (72) has been inverted, (56) can be written as follows:

$$
\begin{aligned}
S_{1}^{I I} & =-\frac{1}{3 c^{D} \gamma}\left(1+j \frac{1}{3 Q_{m} \gamma}\right) \Phi_{o}^{I I} \\
& -\frac{h \epsilon^{s}}{3 c^{D} \gamma}\left(1+j \frac{1}{3 Q_{m} \gamma}\right) \Psi_{o}^{I I} \\
D_{1}^{I I} & =-\frac{h \epsilon^{s}}{3 c^{D} \gamma}\left(1+j \frac{1}{3 Q_{m} \gamma}\right) \Phi_{o}^{I I} \\
& -\frac{\epsilon^{s}}{3 \gamma}\left(1-4 \gamma+j \frac{k^{2}}{3 Q_{m} \gamma}\right) \Psi_{o}^{I I}
\end{aligned}
$$

which are matched with the mechanical displacement and the electrical current at the double frequency, respectively.

The functions $\Phi_{o}^{I I}$ and $\Psi_{o}^{I I}$ are obtained from the Fourier transform of the functions $\Phi_{\mathbf{o}}$ y $\Psi_{\mathbf{o}}$, after they have been calculated from the values of:

$$
\begin{gathered}
\mathbf{D}_{\mathbf{o}}=D_{o} \cos \omega_{o} t \\
\mathbf{S}_{\mathbf{o}}=\frac{D_{o}}{h \epsilon^{s}}\left(\cos \omega_{o} t+\frac{1}{Q_{m}(1-\gamma)} \sin \omega_{o} t\right)
\end{gathered}
$$

where $\mathbf{S}_{\mathbf{o}}$ has been obtained through the inverse Fourier transform of (69).

\section{A Particular Case of a Nonlinear Function}

Let us suppose that nonlinearity is so weak that we can still assume the power series development of the energy function. Then the functions $\Phi$ y $\Psi$ are related because they are derived from the same potential $U$; if $U_{o}$ is the linearized potential energy function, we have:

$$
\begin{aligned}
& \Phi=\frac{\partial U}{\partial \mathbf{S}}-\frac{\partial U_{o}}{\partial \mathbf{S}}=\frac{\partial \Delta U}{\partial \mathbf{S}} \\
& \Psi=\frac{\partial U}{\partial \mathbf{D}}-\frac{\partial U_{o}}{\partial \mathbf{D}}=\frac{\partial \Delta U}{\partial \mathbf{D}}
\end{aligned}
$$

Now we calculate a simple case of nonlinearity described by a quadratic term, which due to its symmetry, can only generate harmonics of frequency $2 \omega$ and 0 (constant term). We wish to calculate the contribution of the elastostrictive term $\Delta U=\lambda_{S S D} \mathbf{S}^{2} \mathbf{D}$ to the second harmonic generated mechanical displacement and electrical current. In this case, both nonlinear functions $\Phi$ and $\Psi$ are not null. From (77) and (78) we obtain:

$$
\Phi_{\mathbf{o}}=2 \lambda_{S S D} \mathbf{S}_{o} \mathbf{D}_{o}
$$

$$
\Psi_{\mathbf{o}}=\lambda_{S S D} \mathbf{S}_{o}^{2}
$$

and substituting (75) and (76) into (79) and (80), we see that:

$$
\begin{aligned}
& \Phi_{\mathbf{o}}=D_{o}^{2} \frac{2 \lambda_{S S D}}{h \epsilon^{s}}\left(\cos ^{2} \omega t+\frac{1}{Q_{m}(1-\gamma)} \sin \omega t \cos \omega t\right) \\
& \Psi_{\mathbf{o}}=D_{o}^{2} \frac{\lambda_{S S D}}{\left(h \epsilon^{s}\right)^{2}}\left(\cos ^{2} \omega t+\frac{2}{Q_{m}(1-\gamma)} \sin \omega t \cos \omega t\right)
\end{aligned}
$$

whose complex amplitudes at frequency $2 \omega_{o}$ are:

$$
\begin{gathered}
\Phi_{o}^{I I}=D_{o}^{2} \frac{\lambda_{S S D}}{h \epsilon^{s}}\left(1-j \frac{1}{Q_{m}(1-\gamma)}\right) \\
\Psi_{o}^{I I}=D_{o}^{2} \frac{\lambda_{S S D}}{2\left(h \epsilon^{s}\right)^{2}}\left(1-j \frac{2}{Q_{m}(1-\gamma)}\right) .
\end{gathered}
$$

By using (83) and (84) in (73) and (74), the first approximation second harmonic generation due to the elastostrictive term can be obtained:

$$
\begin{gathered}
S_{1}^{I I}(S S D)=-D_{o}^{2} \frac{\lambda_{S S D}}{h \epsilon^{s} c^{D}} \frac{1}{2 \gamma}\left(1+j \frac{f_{s}}{Q_{m}}\right) \\
D_{1}^{I I}(S S D)=-D_{o}^{2} \frac{\lambda_{S S D}}{h^{2} \epsilon} \frac{1-2 \gamma}{2 \gamma}\left(1+j \frac{f_{d}}{Q_{m}}\right)
\end{gathered}
$$

where

$$
\begin{gathered}
f_{s}=\frac{1-5 \gamma}{3 \gamma(1-\gamma)} \\
f_{d}=\frac{\gamma}{(1-\gamma)(1-2 \gamma)}
\end{gathered}
$$

It must be pointed out that $\gamma$ can take values near to 0.5 , so (86) must often be rewritten in a convenient form to avoid indetermination.

This method allows the independent evaluation of the contribution of each cubic term of $\Delta U$ to the electrical current and to the mechanical displacement, giving expressions similar to that calculated in the example:

$$
\begin{aligned}
& D_{1}^{I I}(m)=\hat{d}_{m} \lambda_{m} D_{o}^{2} \\
& S_{1}^{I I}(m)=\hat{s}_{m} \lambda_{m} D_{o}^{2}
\end{aligned}
$$

where $m$ refers to any of the four cubic terms. The complex constants $\hat{d}_{m}$ and $\hat{s}_{m}$ are strongly dependent on the electromechanical coupling factor of the employed material.

\section{Conclusions}

With the aim of studying the nonlinear behavior of a piezoelectric resonator, a perturbative method was developed, enabling us to analyze the nonlinear effects in nondistributed systems, on the assumption that there are 
mechanical, electrical, and piezoelectric losses. No restrictions were imposed on the form of the nonlinear function, so the results are valid for a range of amplitudes larger than those in which the power series development of the potential energy is accepted.

The problem can be simplified if the amplitude of the electrical current at fundamental frequency is previously fixed, and the electromotive force is perfectly sinusoidal but of unknown amplitude (so we assume that the generator has no distortion) and there is no mechanical excitation. On this basis, the amplitude and phase of the excitation voltage can be found as a function of the electrical displacement $D_{o}$, as well as the amplitude and phase of the current and mechanical displacement of the generated harmonics.

The nonlinear functions can be split into symmetrical and antisymmetrical parts, and each part has a different influence on the response of the system, depending on whether the first or second approximation is considered. At first order perturbation (direct effect), the antisymmetrical terms produce odd harmonics; the symmetrical terms give even harmonics at first order, but odd harmonics at second order perturbation (indirect effect). When only antisymmetrical terms are significant, the increment of impedance of the resonator is directly related to the fundamental frequency component of the nonlinear functions.

In the actual form, the method cannot be applied to solve subharmonic generation because the solution is developed in harmonics terms, whose frequency is multiple of the excitation one. In order to analyze a given subharmonic, all boundary conditions must be reconsidered, taking the fractional frequency as the fundamental one and applying an electromotive force only at the correct frequency.

There is a unique value of electromotive force for each value of the current. So the resonance hysteresis occurs when, due to the nonlinear character of the equations, a single value of the e.m.f. modulus is assigned to two different values of the current $i$.

Although the current at the excitation frequency is fixed a priori, energy interchange between fundamental and harmonics is not forbidden because the e.m.f. depends on the harmonic frequencies behavior. The energy involved at the fundamental frequency also depends on it.

\section{REFERENCES}

[1] H. F. Tiersten, "Analysis of nonlinear resonance in thicknessshear and trapped energy resonator," J. Acoust. Soc. Amer., vol. 59, pp. 866-878, Apr. 1976.

[2] D. Guyomar, N. Aurelle, C. Richard, P. Gonnard, and L. Eyraud, "Nonlinearities in langevin transducers," in Proc. IEEE Int. Ultrason. Symp., vol. 2, Cannes, France, Nov. 1994, pp. 925928.

[3] H. Beige and G. Schmidt, "Electromechanical resonances for investigating linear and nonlinear properties of dielectric," Ferroelectrics, vol. 41, pp. 39-49, 1982.
[4] R. Pérez and A. Albareda, "Analysis of nolinear effects in a piezoelectric resonator," J. Acoust. Soc. Amer., vol. 100, pp. 3561-3569, Dec. 1996.

[5] R. Pérez, A. Albareda, E. Minguella, and J. L. Villar, "Electrical model for a nonlinear piezoelectric tranducer," in Proc. Tenth IEEE Int. Symp. Appl. Ferroelectr., vol. II, East Brunswick, NJ, Aug., 1996, pp. 955-958.

[6] R. A. Guyer and P. A. Johnson, "Nonlinear mesoscopic elasticity: Evidence for a new class of materials," Phys. Today, pp. 30-36, Apr. 1999.

[7] A. H. Nayfeh, Introduction to Perturbation Techniques, New York: Willey, 1981.

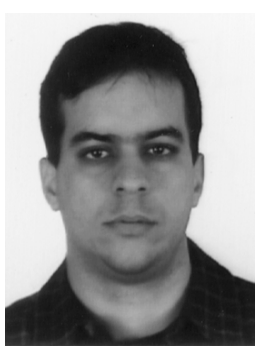

Jose E. García was born in Holguin, Cuba, 1974. He received the physics degree from the University of Oriente, Cuba, in 1996.

During 1997 he worked as a teacher assistant in the Physics Department of the University of Holguin, Cuba. He currently has a Predoctoral Grant in the Department of Applied Physics at the Polytechnic University of Catalonia.

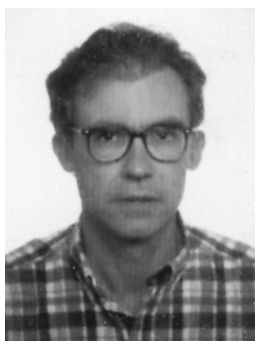

Rafel Pérez was born in Barcelona, Spain, in 1948. He received the Ph.D in physics from the University of Barcelona in 1980

Since 1980 he has been working on ferroelectrical switching and piezoelectrical materials and devices at the Department of Applied Physics at the Polytechnic University of Catalonia, where he has been a professor of physics since 1972 .

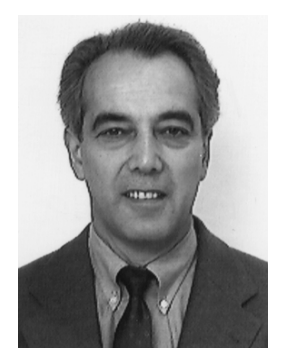

Alfons Albareda was born in Barcelona, Spain, in 1948. He received the Maitrise in physics in 1971 and the Ph.D in physics in 1975 from the University Paul Sabatier (Toulouse, France) and the Ph.D. in physics from the University of Barcelona in 1982.

Since 1989 he has been working on piezoceramic devices and measurement systems at the Department of Applied Physics from the Polytechnic University of Catalonia, Spain, where he has been a professor of communications School. physics since 1977 at the Engineer of Tele-

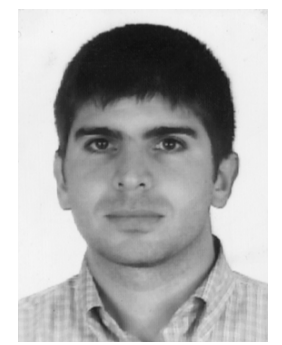

Enric Minguella was born in Barcelona, Spain, in 1967. He received the physics degree from Autonomous University of Barcelona in 1991.

Since 1992 he has been working as a teacher assistant in the Polytechnic University of Catalonia. He currently, is working on nonlinear modeling for power acoustic devices, driver design, and measurement systems in order to obtain the doctoral thesis. 